Denniston, J. C., Chang, R., \& Miller, R. R. (2003). Massive extinction treatment attenuates the renewal effect. Learning and Motivation, 34(1): 68-86. (Feb 2003) Published by Elsevier (ISSN: 1095-9122). DOI:10.1016/S00239690(02)00508-8

\title{
Massive extinction treatment attenuates the renewal effect
}

James C. Denniston, Raymond C. Chang, and Ralph R. Miller

\begin{abstract}
Two experiments with rats as subjects investigated whether massive extinction can attenuate the renewal effect. Experiment 1 investigated whether moderate or massive extinction could prevent the return of conditioned responding following Pavlovian conditioning in Context $A$, extinction in Context $B$, and subsequent testing in Context $C$ (i.e., $A B C$ renewal). Experiment 2 examined whether massive extinction could prevent renewal following training in Context $A$, extinction in Context $B$, and testing in Context $A$ (i.e., ABA renewal). Both experiments observed attenuated renewal following massive, but not moderate extinction. Results are discussed in terms of contemporary theories of extinction.
\end{abstract}


The processes responsible for the experimental extinction of Pavlovian associations have been a focal point of researchers for many years (e.g., Pavlov, 1927). Numerous researchers have reported that, following experimental extinction, behavior indicative of extinction (i.e., weak conditioned responding) tends to be restricted to the context in which the extinction treatment was implemented (e.g., Bouton \& Bolles, 1979; Bouton \& Ricker, 1994; Chelonis, Calton, Hart, \& Schachtman, 1999; Gunther, Denniston, \& Miller, 1998; Lovibond, Preston, \& Mackintosh, 1984; Rauhut, Thomas, \& Ayres, 2001). This observation which is suggestive of the context specificity of extinction, has been termed the renewal effect and has attracted a great deal of attention due to its potential to illuminate the mechanisms underlying experimental extinction and because of its clinical implications (i.e., that exposure-based therapies might be restricted to the place in which the therapy is provided; Bouton and Bouton; Rodriguez, Craske, Mineka, \& Hladek, 1999).

The renewal effect has been demonstrated using three different procedures. One form of renewal occurs when acquisition training is provided in Context $A$, extinction treatment in Context B, and subsequent behavioral testing in Context C (i.e., ABC Renewal; Bouton \& Bolles, 1979). Renewal in an ABC preparation is evidenced by enhanced conditioned responding to the previously extinguished conditioned stimulus (CS) when testing is conducted in Context $\mathrm{C}$, relative to subjects that were tested in the context used for extinction treatment (e.g., Context B). Another form of renewal is that obtained with an ABA design, in which acquisition training is conducted in Context $A$, extinction treatment in Context $B$, and behavioral testing in Context $A$, relative to subjects that receive training, extinction, and testing in the same context (i.e., AAA, Bouton \& Bolles). The third form of renewal is termed AAB renewal, in which acquisition and extinction treatments are both provided in Context $A$, with subsequent behavioral testing in Context B, relative to an AAA control condition (Bouton \& Ricker, 1994). Enhanced conditioned responding to the test CS in Context B demonstrates this form of the renewal effect. One difference between these procedures is the strength of the return of conditioned responding observed at test (as evidenced by cross-experiment comparisons). Specifically, ABA renewal tends to be more robust than either ABC or AAB renewal (Bouton, 1991; Bouton \& Bolles, 1979; Bouton \& King, 1983; Bouton \& Swartzentruber, 1989; Tamai \& Nakajima, 2000).

The mechanism underlying the renewal effect has been a focus of research for several years. One possible explanation of the renewal effect is that the extinction context becomes a conditioned inhibitor as a consequence of the nonreinforced presentations of the excitatory CS in the context (i.e., a form of Pavlovian inhibition). However, numerous studies have failed to find that the extinction context can pass summation and retardation tests for conditioned inhibition (e.g., Bouton \& King, 1983; Bouton \& Swartzentruber, 1986). An alternative interpretation of the renewal effect is that the extinguished CS gains inhibitory strength during extinction. Supportive of such a view are recent studies that have found that massive extinction allows an extinguished CS to pass both summation and retardation tests for conditioned inhibition (e.g., Denniston \& Miller, in press; Hart, Bourne, \& Schachtman, 1995; but see Rauhut et al., 2001, for an exception). Toward explaining the context specificity of extinction (inhibition), Bouton and colleagues (Bouton, 1994; Bouton \& Nelson, 1994; Bouton \& Swartzentruber, 1986) have suggested that the CS becomes ambiguous following extinction treatment as a 
consequence of having signaled both reinforcement and nonreinforcement, and that the context acts as an occasion setter which disambiguates the meaning of the CS. That is, acquisition training establishes an excitatory CS-unconditioned stimulus (US) association, whereas extinction treatment establishes an inhibitory CS-US association (i.e., CS-noUS). This latter association is modulated by the context in which it was acquired, whereas the former excitatory association is not typically modulated by context (see Nelson, 2002, who found that the association that is learned second is relatively context specific). In other words, the context functions as an "AND" gate, in which the joint presence of the CS and the extinction context are necessary for the CS-noUS association to be expressed. When testing is conducted outside of the extinction treatment context, the absence of the context results in decreased activation of the CS-noUS association, thereby allowing for full expression of the excitatory CS-US association (i.e., renewal). The observation that extinction is relatively context specific is consistent with the view that the extinction context functions as a negative occasion setter for the CS-US association, rather than as a conditioned inhibitor.

Recent research has identified circumstances that limit the context specificity of experimental extinction. For example, Gunther et al. (1998) found that, following conditioning in Context A and extinction treatment in Contexts $\mathrm{B}, \mathrm{C}$, and $\mathrm{D}$, renewal of conditioned responding was attenuated when testing was conducted in associatively neutral Context $E$ (note that this is effectively an $A B C$ design). Similar results using a conditioned taste aversion preparation were reported by Chelonis et al. (1999) who found that following taste aversion training in Context $A$ and extinction in Contexts $B, C$, and $D$, renewal was attenuated when testing was conducted in Context A (this was effectively an ABA design). According to the contextual occasion setting account, behavior indicative of extinction would be expected to be restricted to the extinction context. In contrast, weak conditioned responding (indicative of generalization of extinction) was observed by both Gunther et al., who tested in an associatively neutral context (i.e., Context E) and Chelonis et al. who provided testing in the context in which acquisition training had been provided (i.e., Context A). Gunther et al. suggested that generalization of elements from the three extinction contexts $(B, C$, and $D)$ to the test context might result in generalization of the tendency to not respond to the CS, as the test context is likely to have more stimulus elements in common with the three extinction contexts than with the single acquisition context. Such a view is consistent with the contextual occasion setting interpretation provided by Bouton and his colleagues (e.g., Bouton, 1993; Bouton \& Nelson, 1994; Bouton \& Swartzentruber, 1986). However, Chelonis et al. explained their results in terms of encoding variability, in which interference by the CS-noUS association is enhanced following extinction in multiple contexts relative to extinction in a single context, thereby facilitating retrieval of the CS-noUS association outside of the extinction contexts.

In a separate line of research, Denniston and Miller (in press) investigated the informational content of inhibitory associations produced through experimental extinction. Their studies found that a massively extinguished CS passed traditional summation and retardation tests for conditioned inhibition and that the passage of these tests depended upon an equivalency between the temporal relationship between the CS and the US during acquisition training and the temporal relationship between the transfer excitor and the US. They claimed that the potential of an extinguished CS to pass summation and retardation tests for conditioned 
inhibition depended (in part) upon the retrievability of the inhibitory CS-US association. That is, extinction treatment might favor retrieval of the inhibitory CS-US association at the expense of the excitatory CS-US association. When the temporal information content of the inhibitory association matched the information content of the excitatory transfer CS, maximal negative summation was observed. These results suggest that massive extinction treatment might provide another means for favoring retrieval of the inhibitory CS-US association established during experimental extinction.

The present series of experiments investigated whether massive extinction treatment can attenuate the renewal effect. Tamai and Nakajima (2000) found that massive, but not moderate, extinction treatment attenuated $A A B$, but not $A B A$ renewal. In their experiment, Tamai and Nakajima provided rats with various magnitudes of extinction treatments (ranging from 32 to 112 extinction trials) following 24 CS-US acquisition trials, and observed both ABA and AAB renewal after 72 extinction trials, but only $A B A$ renewal following 112 extinction trials. Thus, $A A B$ renewal was attenuated following prolonged extinction treatment. They interpreted their results as being consistent with Bouton and Ricker's (1994) view that the extinction context functions as a negative occasion setter for the inhibitory CS-US association and that conditioned responding is more likely to be "released" when testing is conducted outside of the extinction context. Tamai and Nakajima hypothesized that the contextual gating might weaken following prolonged extinction, thereby providing an explanation for the attenuation of AAB renewal. Toward explaining the preservation of ABA renewal following prolonged extinction treatment, they suggested that the acquisition context $(A)$ can function as a "retrieval cue" for the excitatory CSUS association. This retrieval cue was present during testing for subjects in their ABA renewal group, but not the $A A B$ renewal group, which can potentially explain why renewal was observed in the former group even after prolonged extinction treatment.

The present series of experiments was conceptually similar to that of Tamai and Nakajima's (2000) experiment. However, we investigated whether massive extinction treatment could attenuate $A B A$ and $A B C$ renewal, rather than $A B A$ and $A A B$ renewal. We anticipated that massive extinction treatment provided in a single context should favor retrieval of the inhibitory CS-US association outside of the extinction context. These anticipated results stand in contrast to the view that the context specificity of extinction is the consequence of occasion setting by context (i.e., Bouton, 1993; Bouton \& Nelson, 1994; Bouton \& Swartzentruber, 1986) and facilitated retrieval of excitation by the acquisition context (Tamai \& Nakajima). Indeed, the occasion setting by context view might anticipate that massive extinction treatment should enhance the potential of the context to serve as a negative occasion setter (provided that the potential of a stimulus to serve as a negative occasion setter is monotonically related to number of training trials), thereby enhancing the context specificity of extinction, a result opposite of what we expected (see Tamai \& Nakajima for a discussion of this possibility).

Notably, other researchers have failed to observe an attenuation of renewal with increased extinction experience (e.g., Bouton \& Swartzentruber, 1989; Rauhut et al., 2001; Tamai \& Nakajima, 2000). However, Bouton and Swartzentruber, who provided 84 nonreinforced exposures to a 1-min CS, did not include a group that received fewer extinction trials (notably, the effect of magnitude of extinction treatment was not the focus of their research). Rauhut et 
al., used an $A B A$ renewal procedure and did not equate exposure to the extinction and testing contexts, leaving open the possibility that their failure to observe an attenuation of the renewal effect following 100 2-min exposures to the CS was the result of residual fear of the test (acquisition) context. Although preCS response rates did not differ, subthreshold fear might have influenced their results. Additionally, Rauhut et al.'s assessment of renewal consisted of a comparison of responding to the extinguished CS during the test session in Context $A$ to that observed during the final extinction session (two days earlier) in Context $B$, which might have allowed for some spontaneous recovery to occur. Although Rauhut et al. argued against the potential of spontaneous recovery to undermine their results, this possibility combined with the failure to equate exposure to the extinction and test contexts calls for a more systematic analysis of whether massive extinction might attenuate renewal. The present series of experiments sought to investigate whether truly massive extinction treatment (nearly eight times the amount provided by Tamai \& Nakajima) can attenuate the renewal effect while avoiding some of the limitations cited above.

\section{EXPERIMENT 1: ABC RENEWAL}

Experiment 1 investigated whether massive extinction could prevent the return of conditioned responding observed following Pavlovian training in one context (Context $A$ ), experimental extinction in a second context (Context $\mathrm{B}$ ), and behavioral testing in a third associatively neutral context (Context $\mathrm{C}$; i.e., $\mathrm{ABC}$ renewal). Four groups of rats received training intended to condition fear to a CS, $\mathrm{X}$, in Context $\mathrm{A}$, followed by either massive, moderate, or no extinction experience in Context $B$ (see Table 1). Specifically, following fear conditioning in Context A, Group Ext-Many received 800 nonreinforced presentations of CS X in Context B, Groups ExtMod and Ext-Mod-B received 160 nonreinforced presentations of CS X in Context B, and Group NoExt merely received equivalent exposure to Context $B$. Following extinction treatment, the potential of $\mathrm{CS} X$ to disrupt baseline drinking rates was assessed in an associatively neutral context for Groups NoExt, Ext-Mod, and Ext-Many, or in Context B for Group Ext-Mod-B. If massive extinction enhances the retrievability of CS-noUS associations, rather than enhancing occasion setting of $\mathrm{CS} X$ by the extinction context, then massive extinction should attenuate the renewal effect in Group Ext-Many, relative to Group Ext-Mod. The purpose of including Group Ext-Mod-B was to demonstrate that the magnitude of extinction provided in Group Ext-Mod was sufficient to attenuate conditioned responding when testing is conducted in the extinction context (Context B).

Table 1. Design Summary for Experiment 1

\begin{tabular}{llllll}
\hline Group & Phase 1 & Phase 2 & Reacclimation & Test & Expected \\
\hline NoExt & $(8 \mathrm{X}-\mathrm{US})_{\mathrm{A}}$ & $\left(\mathrm{B}_{\mathrm{B}} / \mathrm{C}_{\mathrm{C}}\right.$ & $O_{\mathrm{B}} / 0_{\mathrm{C}}$ & $(\mathrm{X})_{\mathrm{C}}$ & $\mathrm{CR}$ \\
Ext-Mod & $(8 \mathrm{X}-\mathrm{US})_{\mathrm{A}}$ & $(160 \mathrm{X} \text {-noUS })_{\mathrm{B}} / 0_{\mathrm{C}}$ & $O_{\mathrm{B}} / O_{\mathrm{C}}$ & $(\mathrm{X})_{\mathrm{C}}$ & $\mathrm{CR}$ \\
Ext-Many & $(8 \mathrm{X}-\mathrm{US})_{\mathrm{A}}$ & $(800 \mathrm{X} \text {-noUS })_{\mathrm{B}} / 0_{\mathrm{C}}$ & $O_{\mathrm{B}} / O_{\mathrm{C}}$ & $(\mathrm{X})_{\mathrm{C}}$ & $\mathrm{cr}$ \\
Ext-Mod-B & $(8 \mathrm{X}-\mathrm{US})_{\mathrm{A}}$ & $(160 \mathrm{X} \text {-noUS })_{\mathrm{B}} / 0_{\mathrm{C}}$ & $O_{\mathrm{B}} / 0_{\mathrm{C}}$ & $(\mathrm{X})_{\mathrm{B}}$ & $\mathrm{cr}$ \\
\hline
\end{tabular}


Note. CS X was a 10-s white noise; US was a 0.5-s, 1.0-mA footshock. Subscript letters (A, B, and $C$ ) refer to contexts. During Phase 2, subjects received two sessions per day, one in Context B and the other in Context C, counterbalanced for order. () represents context exposure with no nominal stimulus presentations. Acclimation (not shown) and Reacclimation sessions in Contexts $B$ and $C$ were provided on separate days prior to both Phase 1 and Testing. Subjects in Group Ext-Mod-B were tested for conditioned responding to CS X in Context B. CR = strong responding expected; $\mathrm{CR}=$ weak responding expected.

\section{Method}

\section{Subjects}

The subjects were 24 male and 24 female, experimentally naive, Sprague-Dawley derived rats from our SUNY-Binghamton breeding colony. Body weights ranged from 247-341 g for males and 191-252 $\mathrm{g}$ for females. The animals were individually housed in standard hanging, stainless-steel, wire-mesh cages in a vivarium maintained on a 16:8-h light:dark cycle. All training occurred approximately midway through the light portion of the cycle. Subjects were allowed free access to food in their home cages, whereas access to water was gradually decreased to $10 \mathrm{~min}$ per day prior to the initiation of the experiment. All subjects were handled for $30 \mathrm{~s}$ three times per week from weaning until the initiation of the study. Subjects were randomly assigned to one of four groups $(n s=12)$, counterbalanced for sex.

\section{Apparatus}

Two types of experimental chambers, designated $\mathrm{R}$ and $\mathrm{V}$, were used. Chamber $\mathrm{R}$ was rectangular in shape and measured $22.75 \times 8.25 \times 13.0 \mathrm{~cm}(I \times w \times h)$. The walls and ceiling of the chamber were constructed of clear Plexiglas and the floors consisted of stainless-steel rods measuring $0.48 \mathrm{~cm}$ in diameter, spaced $1.5 \mathrm{~cm}$ center-to-center. The rods were connected by NE2-neon bulbs, which allowed for the delivery of constant-current footshock produced by a high-voltage $A C$ circuit in series with a 1.0-M $\Omega$ resistor. Each of 12 copies of Chamber $R$ was housed in a separate sound- and light-attenuating environmental enclosure. Chamber $\mathrm{R}$ could be dimly illuminated by a 2.0-W (nominal at $120 \mathrm{VAC}$ ) house light driven at $60 \mathrm{VAC}$. The bulb was located on the inside wall of the environmental enclosure, approximately $30 \mathrm{~cm}$ from the center of the experimental chamber.

Chamber $\mathrm{V}$ was a $25.5 \mathrm{~cm}$ long box in the shape of a vertical truncated- $\mathrm{V}$. The chamber was $28 \mathrm{~cm}$ high, $21 \mathrm{~cm}$ wide at the top, and narrowed to $5.25 \mathrm{~cm}$ wide at the bottom. The ceiling was constructed of clear Plexiglas, the front and back end walls were black Plexiglas, and the side walls were stainless steel. The floor consisted of two $25.5 \mathrm{~cm}$ long parallel stainless steel plates, each $2 \mathrm{~cm}$ wide and separated by a 1.25-cm gap. A constant-current footshock could be delivered through the metal walls and floor of the chamber. Each of 12 copies of Chamber $V$ was housed in a separate sound- and light-attenuating enclosure. Chamber $V$ was illuminated by a $7-W$ (nominal at $120 \mathrm{VAC}$ ) bulb driven at $60 \mathrm{VAC}$. The bulb was mounted on the inside wall 
of the environmental enclosure, approximately $30 \mathrm{~cm}$ from the center of the experimental chamber, with the light entering the chamber primarily by reflection from the ceiling of the environmental enclosure. The light intensities in Chambers $\mathrm{R}$ and $\mathrm{V}$ were approximately equal, despite the discrepancy in the light bulbs used, because of the differences between the chambers in the opaqueness of the walls.

Chambers $\mathrm{R}$ and $\mathrm{V}$ could each be equipped with a water-filled lick tube. When inserted, the lick tube extended $1 \mathrm{~cm}$ into a cylindrical drinking recess that was set into one of the Plexiglas end walls of the chamber. Each drinking recess was left-right centered with its bottom $1.75 \mathrm{~cm}$ above the floor of the chamber. The recess was $4.5 \mathrm{~cm}$ in diameter and $5 \mathrm{~cm}$ deep. An infrared photobeam was projected horizontally across the recess, $1 \mathrm{~cm}$ in front of the lick tube. To drink from the lick tube, a subject had to insert its head into the recess, thereby breaking the photobeam. By this means, we could monitor when subjects were accessing the lick tube. A speaker mounted on a wall in each enclosure could deliver a white noise stimulus $8 \mathrm{~dB}$ ( $C$ scale) above the ambient background of $74 \mathrm{~dB}$ ( $\mathrm{C}$ scale), which was produced primarily by a ventilation fan in each environmental enclosure. The white-noise CS was $10 \mathrm{~s}$ in duration and the US was a 0.5-s, 1.0-mA footshock.

Contexts $A, B$, and $C$ were created in the following manner. Context A consisted of the 12 examples of Chamber $\mathrm{R}$ with an odor cue present (one drop of $98 \%$ methyl salicylate on a wooden block inside the environmental enclosure) and the turning off of the house light that otherwise illuminated Chamber R. Context B consisted of Chamber $V$ for half of the subjects in each of the four groups and Chamber $R$ (a different example than had been used for Context $A$ ) for the remaining subjects. This instance of Chamber $\mathrm{R}$ was further differentiated from the chamber that served as Context $A$ through the addition of a clear Plexiglas floor plate that covered the grid floor, the illumination of the house light, and the removal of the odor cue that had been present in Context $A$. Context $C$ was created by switching the type of enclosure ( $R$ or $V)$ from that which served as Context $B$. That is, animals for which a chamber of type $V$ served as Context B were now trained in chamber of type R. Hence, the type of chamber serving as Contexts $\mathrm{B}$ and $\mathrm{C}$ was counterbalanced within groups.

\section{Procedure (see Table 1)}

\section{Acclimation}

Acclimation to the experimental chambers comprising Contexts $B$ and $C$ was conducted on Days 1 and 2 during daily 60-min sessions, counterbalanced within groups for order (B on Day 1 and $C$ on Day 2, or vice versa). Water-filled lick tubes were available and no nominal punctate stimuli were presented. This phase of the experiment served to establish a stable baseline level of drinking behavior, a departure from which would serve as the dependent variable during testing. Following acclimation, the water-filled lick tubes were removed from all chambers. 


\section{Acquisition (Phase 1)}

All subjects received four CS X-US pairings during each daily 60-min session in Context A (Days 3 and 4). During each of these sessions, the footshock US was presented immediately following termination of CS X. Trials were presented at 8, 28, 37, and 55 min into each session.

\section{Extinction (Phase 2)}

All subjects received two daily 120-min sessions on each of four days (Days 5-8). These sessions were separated by $3 \mathrm{~h}$. For Groups Ext-Mod, Ext-Mod-B and Ext-Many, one of these daily sessions consisted of experimental extinction of CS X in Context B. Group No-Ext received equivalent exposure to Context $B$. These extinction sessions consisted of 40 nonreinforced exposures to CS X per session for Groups Ext-Mod and Ext-Mod-B. For these groups, the mean intertrial interval (CS onset to CS onset) was $180 \mathrm{~s}$ (range $=152-208 \mathrm{~s}$ ). Group Ext-Many received 200 nonreinforced presentations of CS X per daily extinction session.[1] For this group, the mean intertrial interval (CS onset to CS onset) was $36 \mathrm{~s}$ (range $=20-52 \mathrm{~s}$ ). The other daily session consisted of exposure to Context $C$ for all subjects. No nominal stimulus was presented during this session. Training session order (extinction or context exposure) was counterbalanced within groups over the four daily sessions using an abba pattern.

\section{Reacclimation}

The lick tubes were returned to the chambers. All subjects were then reacclimated to Contexts $B$ and $C$ on separate days (9 and 10) during daily 60-min sessions (counterbalanced within groups for order). These sessions served to restabilize baseline drinking which might have been disrupted by the footshock USs.

\section{Test}

Testing was conducted during a single 16-min session (Day 11) in either Context B (Group ExtMod-B) or Context C (Groups No-Ext, Ext-Mod, and Ext-Many). During this test session, the animals were allowed to drink from the lick tubes for five cumulative seconds, after which the test stimulus was presented. Thus, all subjects were drinking at the moment of test stimulus onset. The time to complete an additional five cumulative seconds of licking in the presence of CS X was recorded. A 15-min ceiling was imposed on the suppression scores.

Any subject requiring more than $60 \mathrm{~s}$ to complete its first five cumulative seconds of drinking (prior to CS onset), thus exhibiting a reluctance to drink in the test context, was scheduled to be eliminated from the data analysis. In practice, no subjects from this experiment or Experiment 2 met this preCS criterion. Thus, all subjects had high baseline lick rates just prior to test stimulus onset. Prior to statistical analysis, all suppression scores were converted to log (base 10) scores 
to better normalize the within-group distributions, thereby facilitating the use of parametric statistics. An a level of .05 was adopted for all statistical tests.

\section{Results and discussion}

The central finding from this study was that massive extinction experience attenuated conditioned responding in Group Ext-Many at test, relative to Group Ext-Mod. That is, Group Ext-Mod demonstrated strong conditioned responding to CS X at test, indicative of the renewal effect, but Group Ext-Many showed weak conditioned responding to CS X (i.e., massive extinction experience appeared to prevent the renewal effect). The above findings were confirmed with the following statistical analyses.

Analysis of the times to complete five cumulative seconds of drinking prior to the presentation of the CS X (i.e., the preCS scores) was accomplished with a one-way analysis of variance (ANOVA) with group (NoExt, Ext-Mod, Ext-Many, and Ext-Mod-B) as the single factor. This analysis revealed no between-group differences, $F(3,44)=1.25$. Thus, there were no significant differences in baseline performance at the time of testing.

Analysis of the time to complete five cumulative seconds of drinking in the presence of the test CS was accomplished with a one-way ANOVA with Group as the single factor. This analysis revealed a significant main effect of group, $F(3,44)=52.03$. Planned comparisons conducted on the suppression scores (see Fig. 1) revealed greater conditioned suppression in Group Ext-Mod than in Group Ext-Mod-B, $F(1,44)=60.13$, thereby demonstrating the basic renewal effect and that the magnitude of extinction provided to Group Ext-Mod-B was sufficient to attenuate conditioned responding to CS X. The somewhat weaker suppression to CS X observed in Group Ext-Mod relative to Group NoExt, $F(1,44)=7.66, p<.01$, indicates that the renewal effect was not complete. Finally, and of greatest importance, Group Ext-Many suppressed less to CS $X$ than did Group Ext-Mod, $F(1,44)=44.04$, thereby demonstrating that massive extinction experience attenuates the renewal effect. 


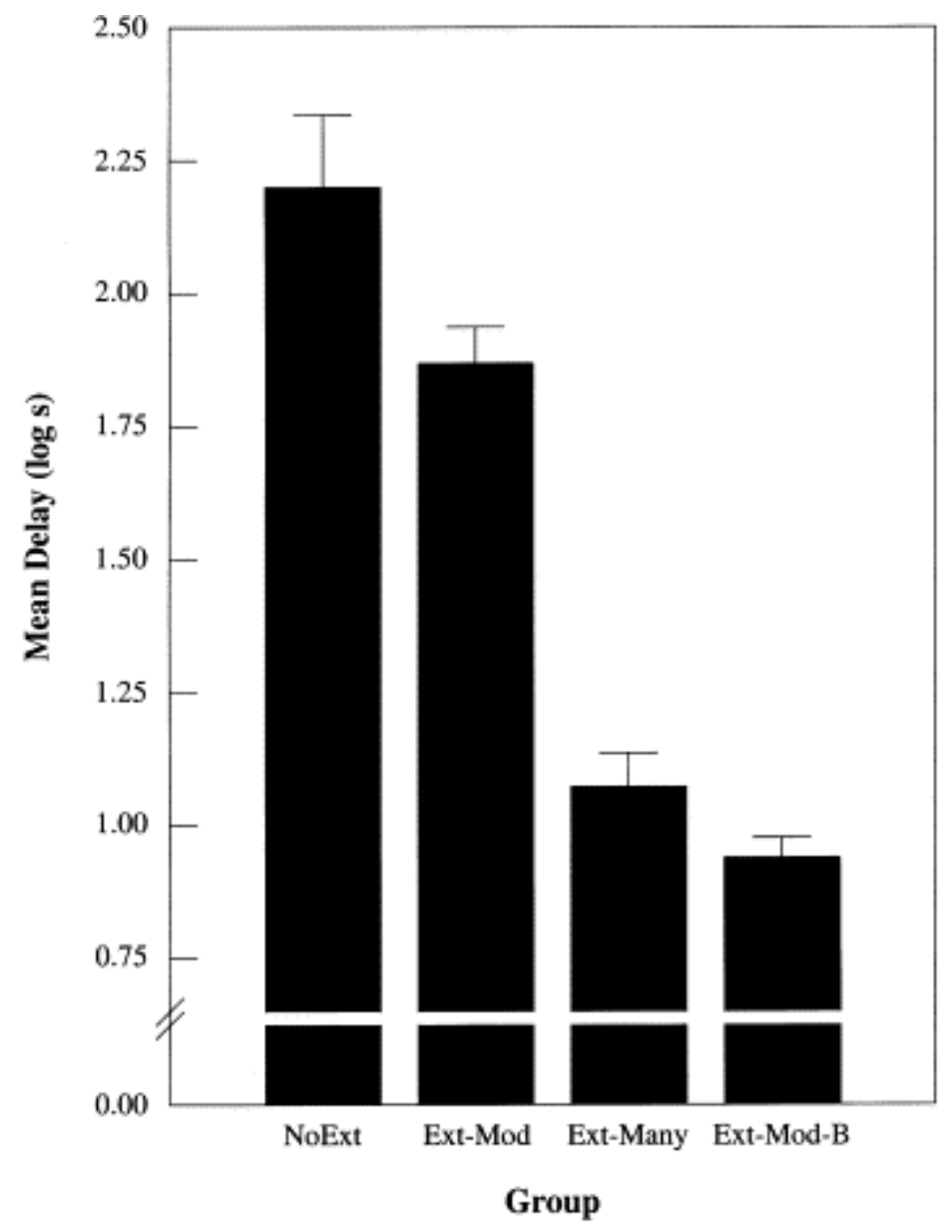

Fig. 1. Experiment 1-ABC Renewal. Group identification refers to the magnitude of extinction experience provided during extinction treatment (no extinction trials, a moderate number of extinction trials, or many extinction trials). Group Ext-Mod-B was tested in the extinction context. Bars depict mean times to complete five cumulative seconds of drinking in the presence of the test stimulus. Greater scores indicate greater fear (i.e., more renewal). Error brackets denote the standard error of the mean. See Table 1 for procedural details.

Thus, Experiment 1 found that, following acquisition training in Context $A$ and moderate extinction experience in Context $\mathrm{B}$, robust conditioned responding (indicative of the renewal effect) was observed when testing was conducted in an associatively neutral context, C (Group Ext-Mod), but not when testing was conducted in the extinction context, B (Group Ext-Mod-B). Of greater importance, massive extinction of CS $X$ attenuated conditioned responding (i.e., reduced the renewal effect) when behavioral testing was conducted in Context C (Group ExtMany). This finding suggests that massive extinction treatment enhances generalization of extinction to other contexts (this point is elaborated in the General Discussion). 


\section{EXPERIMENT 2: ABA RENEWAL}

Experiment 1 found that massive extinction attenuates the return of conditioned responding observed when testing is conducted in an associatively neutral context (i.e., ABC renewal). The purpose of Experiment 2 was to investigate whether a similar result would be obtained following acquisition training in Context $A$, extinction training in Context $B$, and behavioral testing in Context A (i.e., ABA renewal). Prior research has demonstrated that ABA renewal tends to be more robust than other forms of renewal (e.g., ABC or AAB; see Bouton, 1991; Tamai \& Nakajima, 2000). Thus, the effectiveness of extensive extinction treatment in thwarting renewal might be limited to instances in which the renewal effect is less robust, such as ABC renewal as in Experiment 1. Therefore, the present experiment sought to investigate whether massive extinction treatment can prevent the robust renewal typically observed as a consequence of behavioral testing in the context in which excitatory conditioning was conducted (Context $A$ ).

The design of Experiment 2 is outlined in Table 2. As in Experiment 1, rats received acquisition training in Context $A$, which consisted of $8 \mathrm{X}$-US pairings. Following acquisition training, subjects in Group Ext-Mod received moderate extinction treatment (160 X-noUS trials) in Context B, subjects in Group Ext-Many received massive extinction treatment (800 X-noUS trials) in Context $B$, subjects in Group Ext-Mod-A received moderate extinction treatment (160 $X$-noUS trials) in Context $A$, and subjects in Group NoExt merely received exposure to the two contexts during extinction treatment. As in Experiment 1, all subjects received equivalent exposure to Contexts $A$ and $B$ during extinction treatment. At test, the potential of CS X to disrupt baseline drinking in Context A was assessed. We expected to observe strong conditioned responding to CS X in Groups NoExt and Ext-Mod, the latter showing the renewal effect. In contrast, we anticipated weak conditioned responding in Group Ext-Mod-A, thereby demonstrating that 160 extinction trials is sufficient to eliminate conditioned responding when behavioral testing is conducted in the same context as that in which acquisition and extinction treatment has been provided, and Ext-Many, demonstrating that massive extinction treatment attenuates the renewal effect.

Table 2. Design Summary for Experiment 2

\begin{tabular}{llllll}
\hline Group & Phase 1 & Phase 2 & Reacclimation & Test & Expected \\
\hline NoExt & $(8 \mathrm{X}-\mathrm{US})_{\mathrm{A}}$ & $\left(\mathrm{O}_{\mathrm{B}} /()_{\mathrm{A}}\right.$ & ()$_{\mathrm{A}}$ & $(\mathrm{X})_{\mathrm{A}}$ & $\mathrm{CR}$ \\
Ext-Mod & $(8 \mathrm{X}-\mathrm{US})_{\mathrm{A}}$ & $(160 \mathrm{X}-\mathrm{noUS})_{\mathrm{B}} / \mathrm{O}_{\mathrm{A}}$ & ()$_{\mathrm{A}}$ & $(\mathrm{X})_{\mathrm{A}}$ & $\mathrm{CR}$ \\
Ext-Many & $(8 \mathrm{X}-\mathrm{US})_{\mathrm{A}}$ & $\left.(800 \mathrm{X}-\text { noUS })_{\mathrm{B}} /\right)_{\mathrm{A}}$ & ()$_{\mathrm{A}}$ & $(\mathrm{X})_{\mathrm{A}}$ & $\mathrm{cr}$ \\
Ext-Mod-A & $(8 \mathrm{X}-\mathrm{US})_{\mathrm{A}}$ & $(160 \mathrm{X}-\text { noUS })_{\mathrm{A}} / \mathrm{O}_{\mathrm{B}}$ & ()$_{\mathrm{A}}$ & $(\mathrm{X})_{\mathrm{A}}$ & $\mathrm{cr}$ \\
\hline
\end{tabular}

Note. CS X was a 10-s white noise; US was a 0.5-, 1.0-A footshock. Subscript letters (A and B) refer to contexts. During Phase 2, subjects received two sessions per day, one in Context $B$ and the other in Context A, counterbalanced for order. () represents context exposure with no nominal stimulus presentations. A single acclimation (not shown) and reacclimation session in 
Context A was provided prior to both Phase 1 and Testing. CR = strong responding expected; $\mathrm{CR}=$ weak responding expected.

\section{Subjects and apparatus}

The subjects were 24 male (259-412 g) and 24 female (200-260 g), 80 to 120-day-old, naïve Sprague-Dawley descended rats from our SUNY-Binghamton breeding colony. Subjects were maintained and housed as in Experiment 1 . Subjects were randomly assigned to one of four groups (ns=12), counterbalanced for sex. The apparatus was the same as in Experiment 1 , except that the houselights were normally on, and the Plexiglas floor plates and odor cues were not necessary for the present experiment because only two contexts were needed for the $A B A$ design (Chambers $R$ and $V$ were used). The types of chamber serving as Contexts $A$ and $B$ were counterbalanced within groups.

\section{Procedure (see Table 2)}

\section{Acclimation}

Acclimation to Context A was conducted on Day 1 during a 60-min session as in Experiment 1.

\section{Acquisition (Phase 1)}

On Days 2 and 3, all subjects received four CS X-US pairings during each daily 60-min session in Context $A$ as in Experiment 1.

\section{Extinction (Phase 2)}

Extinction training was conducted on Days 4-7 as in Experiment 1. Subjects in Group Ext-Mod and Ext-Mod-A received 40 nonreinforced exposures to CS X per session in Contexts B and A, respectively. Trial spacing was the same as in Experiment 1. Subjects in Group Ext-Many received treatment identical to that provided in Experiment 1 (i.e., 200 nonreinforced presentations of CS X per daily extinction session). Subjects in Group NoExt received equivalent exposure to Context $\mathrm{B}$, with no nominal stimulus presentations. As in Experiment 1, a second daily session consisted of exposure to Context A for subjects in Groups Ext-Mod, ExtMany, and NoExt, and to Context B for subjects in Group Ext-Mod-A. Training session order (extinction or context exposure) was counterbalanced within groups over the four daily sessions using an abba pattern. 


\section{Reacclimation}

The lick tubes were returned to the chambers. All subjects were then reacclimated to the experimental context (A) during a single 60-min session (Day 8), in order to restabilize baseline drinking which might have been disrupted by the footshock USs. A single reacclimation session was provided because we observed little disruption of baseline drinking in Experiment 1 following the four sessions of context exposure during Phase 2.

\section{Test}

Testing was conducted during a single 16-min session (Day 9) in Context A following the same procedure that was used in Experiment 1. Prior to statistical analysis, all suppression scores were converted to log (base 10) scores as in Experiment 1.

\section{Results and discussion}

The results of Experiment 2 replicate those of Experiment 1, but now in an ABA renewal procedure. That is, the massive extinction experience provided to Group Ext-Many in Context B thwarted the return of conditioned responding when behavioral testing was conducted in Context A relative to Group Ext-Mod, which demonstrated strong conditioned responding to CS

$X$ (indicative of the renewal effect) at test. The above findings were confirmed with the following statistical analyses.

As in Experiment 1, we first analyzed the times to complete five cumulative seconds of drinking prior to the presentation of CS X (i.e., the preCS scores) with a one-way AVOVA with group (NoExt, Ext-Mod, Ext-Many, and Ext-Mod-A) as the single factor. This analysis revealed no between-group differences, $F(3,44)=1.53$. Thus, there were no significant differences in baseline performance at the time of testing.

Analysis of the time to complete five cumulative seconds of drinking in the presence of the test CS was accomplished with a one-way ANOVA with group as the single factor. This analysis revealed a main effect of group, $F(3,44)=24.53$. Planned comparisons conducted on the suppression scores (see Fig. 2) revealed greater conditioned suppression in Group Ext-Mod than in Group Ext-Mod-A, $F(1,44)=21.55$, thereby demonstrating the basic renewal effect and that the magnitude of extinction provided to Group Ext-Mod-A was sufficient to attenuate conditioned responding to CS X. The somewhat weaker suppression to CS X observed in Group Ext-Mod relative to Group NoExt, $F(1,44)=6.92, p<.05$, indicates that the renewal effect was not complete. Finally, and of greatest importance, Group Ext-Many suppressed less to CS $X$ than did Group Ext-Mod, $F(1,44)=18.17$, thereby demonstrating that massive extinction experience attenuates the renewal effect. 


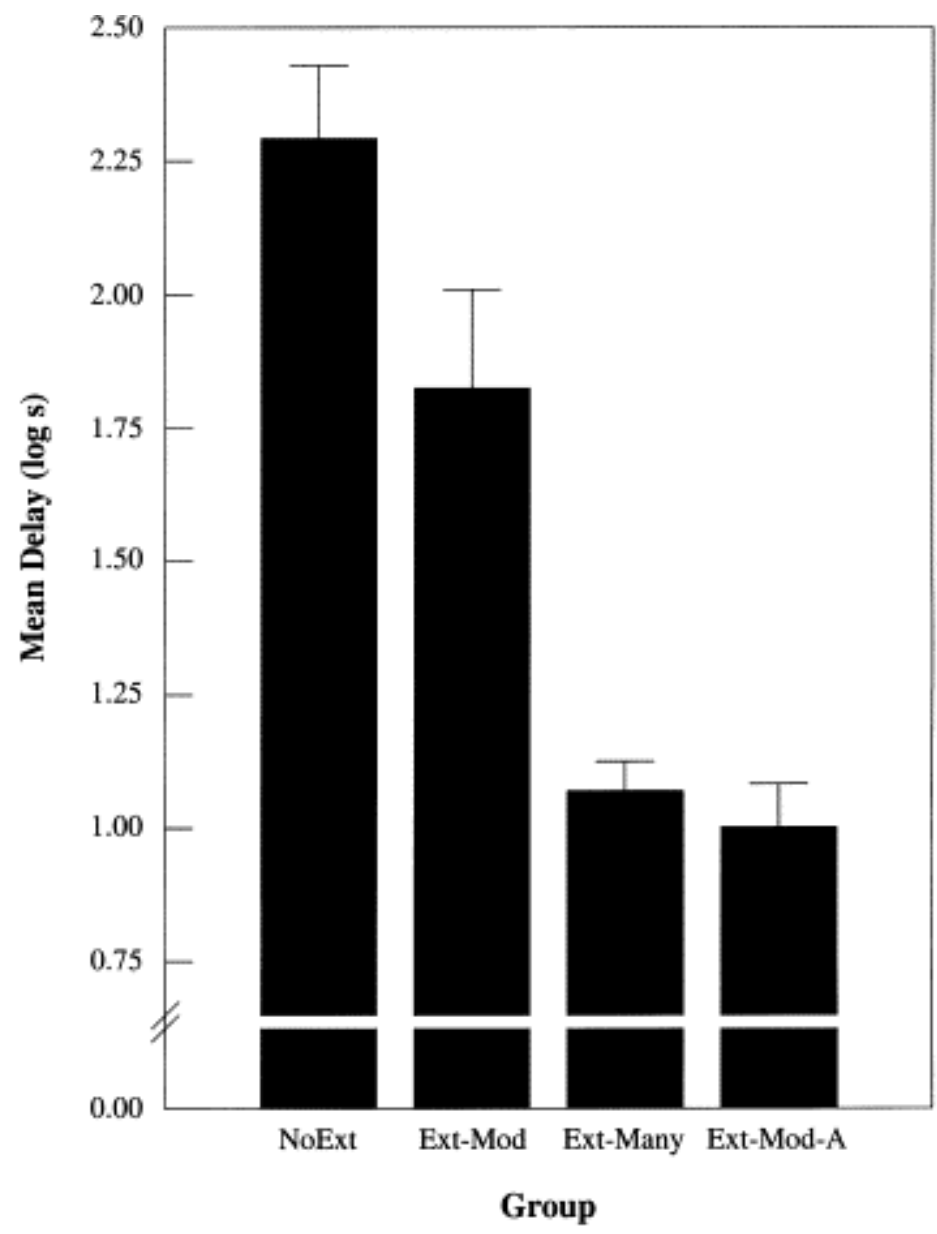

Fig. 2. Experiment 2-ABA Renewal. Group identification refers to the magnitude of extinction experience provided during extinction treatment (no extinction trials, a moderate number of extinction trials, or many extinction trials). Group Ext-Mod-A was tested in the extinction context. Bars depict mean times to complete five cumulative seconds of drinking in the presence of the test stimulus. Greater scores indicate greater fear (i.e., more renewal). Error brackets denote the standard error of the mean. See Table 1 for procedural details.

Thus, Experiment 2 found that following acquisition training in Context $A$ and moderate extinction experience in Context $\mathrm{B}$, robust renewal of conditioned responding was observed when testing was conducted in Context A (Group Ext-Mod), but not when both extinction treatment and testing were conducted in Context A (Group Ext-Mod-A). This latter result confirms that 160 extinction trials were sufficient to produce extinction. Thus, the strong conditioned responding to CS X in Group Ext-Mod presumably reflects the renewal effect, rather than insufficient extinction treatment. Most centrally, massive extinction of CS X in Context B attenuated the return of conditioned responding when behavioral testing was conducted in Context $A$. That is, massive extinction treatment attenuated ABA renewal, suggesting that massive extinction treatment enhances generalization of extinction experience across contexts, 
even when behavioral testing is conducted in the same context as that in which excitatory training was provided (a condition which ordinarily tends to enhance renewal).

\section{GENERAL DISCUSSION}

Experiment 1 demonstrated that massive, relative to moderate extinction treatment, can attenuate the renewal effect when acquisition training is provided in Context $A$, extinction treatment in Context $B$, and behavioral testing in Context $C$ (i.e., ABC renewal). Similarly, Experiment 2 demonstrated that massive, relative to moderate extinction treatment, can attenuate the renewal effect when behavioral testing was in the context in which acquisition training was provided (Context A; i.e., ABA renewal, which ordinarily produces more robust renewal than the $A B C$ procedure). These results suggest that massive extinction treatment allows for the expression of extinction outside of the extinction context.

Numerous researchers have claimed that experimental extinction is the result of an inhibitory process that counters the excitation established during acquisition treatment (e.g., Bouton \& Nelson, 1994; Calton, Mitchell, \& Schachtman, 1996; Denniston \& Miller, in press; Pavlov, 1927; Rescorla, 1996). However, the context specificity of extinction requires further explanation. One explanation is in terms of occasion setting by the extinction context (Bouton \& Nelson) in which nonreinforcement of the CS in the extinction context results in that context serving as a negative occasion setter for the CS-US association. That is, the CS becomes ambiguous as a consequence of prior reinforcement and nonreinforcement, and the context acts to disambiguate the potential meanings of the CS. Hence, behavior indicative of extinction should be restricted to the extinction context because joint presence of the CS and the context is necessary for expression of the inhibitory CS-US association. An alternative view is that inhibitory associations are more transient and generalize less than excitatory associations ( Konorski, 1948; Pavlov, 1927; Spence, 1937). According to this view, inhibitory associations have narrower generalization gradients than excitatory associations and as a consequence might be less able to generalize across contexts relative to excitatory associations.

The observation that extinction in multiple contexts can attenuate the renewal effect (relative to the same amount of extinction treatment in a single context, Chelonis et al., 1999; Gunther et al., 1998) is compatible with either extinction producing inhibition with narrow generalization gradients or occasion setting by context because both views would anticipate that a (nonextinction) test context would have more stimulus elements in common with many extinction contexts than with a single extinction context. However, massive extinction in one context, as examined in the present research, differentiates between these two accounts because massive extinction might broaden the resultant inhibitory generalization gradient (provided that the inhibitory association is relatively stronger following massive extinction treatment than moderate extinction treatment). In contrast, occasion setting of the CS-noUS association should be better learned with more extinction trials, thereby adding to the context specificity of extinction. That is, massive extinction treatment should have strengthened the occasion setting potential of the context, thereby enhancing the context specificity of extinction, a result the opposite of that observed in the present experiments. Instead, we posit that massive 
extinction enhances the retrievability of an inhibitory association that is presumably established during extinction treatment, thereby allowing for expression of the inhibitory association outside of the extinction context. Supportive of this view, we observed the typical renewal effect following moderate, but not massive extinction treatments in both Experiments 1 and 2 . Importantly, we are not calling into question the encoding variability account of Chelonis et al.'s results (i.e., that extinction in multiple contexts results in greater encoding variability, thereby facilitating retrieval of the inhibitory association outside of the extinction contexts). Rather, we suggest that massive extinction treatment is a means to a similar end, that is, enhanced retrieval of the inhibitory association outside of the context(s) in which extinction treatment was provided.

Another potential explanation of our results is that they do not reflect either inhibitory processes or negative occasion setting. Recently, Aguado, de Brugada, and Hall (2001) reported that an extinguished CS could pass both summation and retardation tests for conditioned inhibition, but the potential of the CS to do so did not reflect conditioned inhibition. They argued that the apparent passage of a negative summation test for conditioned inhibition merely reflected stimulus generalization decrement with respect to the transfer excitor, whereas the apparent passage of a retardation test for conditioned inhibition reflected the same type of decrease in attention that is often thought to underlie latent inhibition. Thus, the weak conditioned responding observed in Groups Ext-Many in both Experiments 1 and 2 might reflect latent inhibition, rather than conditioned inhibition, generalizing to a new context. However, there are a few potential problems with such an explanation of our results. First, there were several limitations to Aguado et al.'s procedure (see Denniston \& Miller, in press, for a discussion). For example, Aguado et al. interspersed extinction of their flavor CS with exposure to a neutral CS during extinction treatment, potentially resulting in the acquisition of inhibition to the neutral CS (and perhaps this protected the experimentally extinguished CS from extinction) and also failed to equate exposure to the extinguished CS and the exposed neutral flavor. Thus, their finding that extinction is the result of attentional rather than inhibitory processes is not conclusive. Moreover, it is implausible that a cue commanding reduced attention would still be able to produce appreciable generalization decrement relative to the transfer excitor of the summation test.

Nevertheless, it is possible that the present prevention of renewal resulting from massive extinction treatment reflects a form of attenuated attention to the CS, especially given the massed nature (i.e., close stimulus spacing) of our massive extinction treatment. That is, we provided massive extinction treatment followed by testing and observed weak conditioned responding despite the test occurring in a different context. Our procedure was similar to that of Aguado et al. (2001), who, following massive extinction treatment, observed retardation of reacquisition of behavioral control (i.e., weak conditioned responding). Thus, despite the difference in test procedure, the results of both series of experiments could potentially be explained by means of decreased attention. However, two empirical results challenge the veracity of this alternative explanation of our data. First, the attentional deficit that is sometimes assumed to underlie the CS-preexposure effect is relatively context specific (e.g., Channell \& Hall, 1983; Lovibond et al., 1984; Lubow, 1989). Second, at both the theoretical and empirical levels, there is good reason to question whether the CS-preexposure effect is the result of an 
attentional process. For example, both Wagner's (1981) SOP model and Miller and Matzel's (1988) comparator hypothesis suggest that the CS-preexposure effect is the result of a strong CS-context association, which interferes with the acquisition (Wagner) or expression (Miller \& Matzel) of the CS-US association, rather than decreased attention to the CS. Supportive of these views is the common observation that a context switch between CS preexposure and subsequent CS-US pairings attenuates the CS-preexposure effect (see above). Consistent with this view, context extinction following stimulus preexposure and subsequent CS-US pairings attenuates the CS-preexposure effect (e.g., Grahame, Barnet, Gunther, \& Miller, 1994), indicating that CS-preexposure is the consequence of a strong CS-context association interfering with expression of the CS-US association. Thus, the present results do not appear to be merely the result of decreased processing of the CS. Instead we suggest that they are due to the acquisition of an inhibitory CS-US association that generalized outside of the extinction context.

The present series of experiments found that massive extinction treatment prevents the renewal effect. These results challenge the interpretations of studies by Bouton and Swartzentruber (1989), Rauhut et al. (2001), and Tamai and Nakajima (2000), who failed to find such an effect (notably Tamai \& Nakajima observed an attenuation of $A A B$, but not $A B A$, renewal following massive extinction treatment). However, the present series provided far more extinction experience than did any of the previously mentioned studies (i.e., nearly eight times the number of extinction trials) and provided the massive extinction treatment in a massed manner. Additionally, we equated exposure to the extinction and test contexts, compared renewal following massive extinction to a group that received moderate extinction, and made these comparisons during parallel test sessions (design features in common with Tamai \& Nakajima's experiment). However, our present results are similar to other aspects of Rauhut et al.'s results. For example Rauhut et al. found that subjecting an excitatory CS to either of three inhibitory procedures (explicitly unpaired, Pavlovian discriminative, or differential inhibition) attenuated the renewal effect. Here we suggest that truly massive extinction experience, using parameters shown to establish the extinguished CS as a conditioned inhibitor, can produce similar results. The findings of Rauhut et al. as well as the present series of experiments add to the repertoire of means of preventing renewal (i.e., extinction in multiple contexts; Chelonis et al., 1999; Gunther et al., 1998). The results of the present series of experiments, as well as those of Chelonis et al., Gunther et al., and Tamai and Nakajima's observed attenuation of AAB renewal, support the view that experimental extinction is the result of the acquisition of an inhibitory CSUS association that interferes with the expression of the excitatory CS-US association. Here we propose that massive extinction enhances retrieval of the inhibitory association outside of the extinction context, which complements Chelonis et al.'s suggestion that extinction treatment in multiple contexts enhances encoding variability, thereby allowing for enhanced retrieval of the inhibitory association outside of the extinction context.

The collective results of the present series of experiments and those of Chelonis et al. (1999), Gunther et al. (1998), Rauhut et al. (2001), and Tamai and Nakajima (2000) have potentially important clinical implications. Therapists seeking to minimize relapse following exposure based therapies might well consider providing: (1) explicitly unpaired exposure to the CS and the US (see Rauhut et al.); (2) extinction treatment in a variety of contexts; or (3) overtraining of 
extinction in a single context. It is likely that a combination of these procedures will be of even greater effectiveness than any one alone.

\section{NOTE}

1. Groups Ext-Many and Ext-Mod differed not only in magnitude of extinction treatment, but also trial spacing during extinction treatment. That is, Group Ext-Many received massed, massive extinction, whereas the moderate extinction treatment groups (Ext-Mod and Ext-Mod-B) received spaced, moderate extinction treatment. This difference in trial spacing was introduced for pragmatic reasons (i.e., to minimize the duration of Phase 2 of the experiment). Notably, Gallistel and Gibbon (2000) have suggested that trial spacing has little effect on experimental extinction.

\section{ACKNOWLEDGEMENTS}

Support for this research was provided by National Institute of Mental Health Grant 33881. We thank James Esposito for his technical support and Jeffrey Amundson, Francisco Arcediano, Martha Escobar, Steven Stout, and Daniel Wheeler for comments on an earlier version of this manuscript.

\section{REFERENCES}

Aguado et al., 2001. L. Aguado, I. de Brugada and G. Hall , Tests for inhibition after extinction of a conditioned stimulus in the flavour aversion procedure. Quarterly Journal of Experimental Psychology 54B (2001), pp. 201-218.

Bouton, 1988. M.E. Bouton, Context and ambiguity in the extinction of emotional learning: Implications for exposure therapy. Learning and Motivation 26 (1988), pp. 137-149.

Bouton, 1991. M.E. Bouton , A contextual analysis of fear extinction. In: P.R. Marin, Editor, Handbook of behavior therapy and psychological science: An integrative approach, Pergamon Press, New York (1991), pp. 435-453.

Bouton, 1993. M.E. Bouton, Context, time, and memory retrieval in the interference paradigms of Pavlovian learning. Psychological Bulletin 114 (1993), pp. 80-99.

Bouton, 1994. M.E. Bouton, Context, ambiguity, and classical conditioning. Current Directions in Psychological Science 3 (1994), pp. 49-53.

Bouton and Bolles, 1979. M.E. Bouton and R.C. Bolles, Contextual control of the extinction of conditioned fear. Learning and Motivation 10 (1979), pp. 445-466. 
Bouton and King, 1983. M.E. Bouton and D.A. King, Contextual control of the extinction of conditioned fear: tests for associative value of the context. Journal of Experimental Psychology: Animal Behavior Processes 9 (1983), pp. 248-265.

Bouton and Nelson, 1994. M.E. Bouton and J.B. Nelson, Context-specificity of target versus feature inhibition in a feature negative discrimination. Journal of Experimental Psychology: Animal Behavior Processes 20 (1994), pp. 51-65.

Bouton and Ricker, 1994. M.E. Bouton and S.T. Ricker, Renewal of extinguished responding in a second context. Animal Learning \& Behavior 22 (1994), pp. 317-324.

Bouton and Swartzentruber, 1986. M.E. Bouton and D. Swartzentruber, Analysis of the associative and occasion-setting properties of contexts participating in a Pavlovian discrimination. Journal of Experimental Psychology: Animal Behavior Processes 12 (1986), pp. 333-350.

Bouton and Swartzentruber, 1989. M.E. Bouton and D. Swartzentruber, Slow reacquisition following extinction: Context, encoding, and retrieval mechanisms. Journal of Experimental Psychology: Animal Behavior Processes 15 (1989), pp. 43-53.

Calton et al., 1996. J.L. Calton, K.G. Mitchell and T.R. Schachtman, Conditioned inhibition produced by extinction of a conditioned stimulus. Learning and Motivation 27 (1996), pp. 335361.

Channell and Hall, 1983. S. Channell and G. Hall , Contextual effects in latent inhibition with an appetitive conditioning procedure. Animal Learning \& Behavior 11 (1983), pp. 67-74.

Chelonis et al., 1999. J.J. Chelonis, J.L. Calton, J.A. Hart and T.R. Schachtman , Attenuation of the renewal effect by extinction in multiple contexts. Learning and Motivation 30 (1999), pp. 114.

Denniston and Miller, in press. Denniston, J. C., \& Miller, R. R. (in press). Temporal specificity of inhibition produced through extinction. Animal Learning \& Behavior.

Gallistel and Gibbon, 2000. C.R. Gallistel and J. Gibbon , Time, rate, and conditioning. Psychological Review 107 (2000), pp. 289-344.

Grahame et al., 1994. N.J. Grahame, R.C. Barnet, L.M. Gunther and R.R. Miller , Latent inhibition as a performance deficit resulting from CS-context associations. Animal Learning \& Behavior 22 (1994), pp. 395-408.

Gunther et al., 1998. L.M. Gunther, J.C. Denniston and R.R. Miller, Conducting exposure treatment in multiple contexts can prevent relapse. Behaviour Research and Therapy 36 (1998), pp. 75-91. 
Hart et al., 1995. J.A. Hart, M.J. Bourne and T.R. Schachtman, Slow reacquisition of a conditioned taste aversion. Animal Learning \& Behavior 23 (1995), pp. 297-303.

Konorski, 1948. J. Konorski, Conditioned reflexes and neural organization. , Cambridge University Press, Cambridge, UK (1948).

Lovibond et al., 1984. P.F. Lovibond, G.C. Preston and N.J. Mackintosh, Context specificity of conditioning, extinction, and latent inhibition. Journal of Experimental Psychology: Animal Behavior Processes 10 (1984), pp. 360-375.

Lubow, 1989. R.E. Lubow, Latent inhibition and conditioned attention theory. , Cambridge University Press, Cambridge, UK (1989).

Miller and Matzel, 1988. R.R. Miller and L.D. Matzel, The comparator hypothesis: A response rule for the expression of associations. In: G.H. Bower, Editor, The psychology of learning and motivation vol. 22, Academic Press, San Diego, CA (1988), pp. 51-92.

Nelson, 2002. J.B. Nelson, Context specificity of excitation and inhibition in ambiguous stimuli. Learning and Motivation 33 (2002), pp. 284-310.

Pavlov, 1927. I.P. Pavlov, Conditioned reflexes., Oxford University Press, London (1927).

Rauhut et al., 2001. A.S. Rauhut, B.L. Thomas and J.J.B. Ayres, Treatments that weaken Pavlovian conditioned fear and thwart its renewal in rats: Implications for treating human phobias. Journal of Experimental Psychology: Animal Behavior Processes 27 (2001), pp. 99114.

Rescorla, 1996. R.A. Rescorla, Preservation of Pavlovian associations through extinction. Quarterly Journal of Experimental Psychology 49B (1996), pp. 245-258.

Rodriguez et al., 1999. B.I. Rodriguez, M.G. Craske, S. Mineka and D. Hladek, Contextspecificity of relapse: Effects of therapist and environmental context on return of fear. Behaviour Research and Therapy 37 (1999), pp. 845-862.

Spence, 1937. K.W. Spence, The differential response in animals to stimuli varying within a single dimension. Psychological Review 44 (1937), pp. 430-444.

Tamai and Nakajima, 2000. N. Tamai and S. Nakajima, Renewal of formerly conditioned fear in rats after extensive extinction training. International Journal of Comparative Psychology 13 (2000), pp. 137-147.

Wagner, 1981. A.R. Wagner, SOP: A model of automatic memory processing in animal behavior. In: N.E. Spear and R.R. Miller, Editors, Information processing in animals: Memory mechanisms, Erlbaum, Hillsdale, NJ (1981), pp. 5-47. 